\title{
P03-224
}

\section{MELATONIN IN TREATMENT OF CHRONIC SLEEP DISORDERS IN ADULTS WITH PERVASIVE DEVELOPMENT DISORDERS: A RETROSPECTIVE STUDY}

N. Deriaz ${ }^{1}$, G. Galli-Carminati ${ }^{2}$, G. Bertschy ${ }^{2}$

${ }^{1}$ Psychiatry, University Hospital of Geneva, Chêne-Bourg, ${ }^{2}$ Psychiatry, University Hospital of Geneva, Geneva, Switzerland

Background: Melatonin may be used to treat sleep disorders in both children and adults with intellectual disability. The evidence for its efficacy, potential adverse effects and drug interactions are reviewed in the context of prescribing to people with intellectual disability.

Methods: This study presents the use of melatonin to treat severe circadian sleep-wake disturbances in 6 adults with pervasive developmental disorders. Melatonin was initiated at a daily dose of $3 \mathrm{mg}$ at nocturnal bedtime. If this proved ineffective, the melatonin dose was titrated over the following 4 weeks at increments of $3 \mathrm{mg} / 2$ weeks up to a maximum of $9 \mathrm{mg}$, unless it was tolerated. Assessments included the Clinical Global Impression-Severity (CGI-S) and CGI-Improvement (CGI-I).

Results: Melatonin administered in the evening dramatically improved the sleep-wake pattern in all patients. Melatonin appears to be effective in reducing sleep onset latency and is probably effective in improving nocturnal awakenings and total sleep time in adults with pervasive developmental disorders. Its effectiveness remained stable for the 6-months period of administration. Melatonin was well-tolerated in all patients and no side effects were noted during the therapy.

Conclusions: Melatonin appears to be promising as an efficient and seemingly safe alternative for treatment of severe circadian sleep disturbances in adults with intellectual disability. There may be heterogeneity of response depending on the nature of the sleep problem and cause of the intellectual disability or associated disabilities. Further studies are necessary before firm conclusions can be drawn and guidelines for the use of melatonin for people with ID formulated. 